The Astrophysical Journal, 226:L115-L118, 1978 December 15

(c) 1978. The American Astronomical Society. All rights reserved. Printed in U.S.A.

\title{
A NEW TYPE OF GALAXY WITH PROLATE STRUCTURE
}

\author{
Francesco Bertola and Giuseppe Galletta \\ Asiago Astrophysical Observatory, University of Padua \\ Received 1978 June 29; accepted 1978 September 6
}

\begin{abstract}
A morphological study of five galaxies has revealed that they are characterized by a dust lane crossing a luminous elliptical-like body along the minor axis. The galaxies are NGC 1947, 5128, and 5363 and those associated with Cyg A and PKS 1934-63. The suggestion is made that these are members of a new class of galaxy with prolate stellar structure cut equatorially by a gaseous plane. The dynamical behavior of these systems is complex and not yet understood.
\end{abstract}

Subject headings: galaxies: internal motions - galaxies: structure

\section{INTRODUCTION}

The considerations which are the subject of this Letter arose from a morphological and spectroscopic study of the galaxy NGC 5363. Originally this object had been included in our observational program of Irr II galaxies, since it is often classified in this way (Holmberg 1958; de Vaucouleurs, de Vaucouleurs, and Corwin 1976). On the Palomar Sky Survey prints NGC 5363 appears as an elliptical galaxy. Nevertheless, very interesting morphology of the inner parts of NGC 5363 was revealed when a 100 inch $(2.54 \mathrm{~m})$ Mount Wilson photograph $(\mathrm{H} 369 \mathrm{H})$ was examined. A very distinct and thin dust lane lies exactly along the minor axis, dividing the body of the galaxy into two parts, of which the NW is the brighter. A star image, which appears slightly elongated in Figure 1 (Plate L10) because of Coma, is superposed on the SW side.

We have been particularly impressed by the quite remarkable similarity of the inner structure of NGC 5363 to the well-known galaxy NGC 5128 (Cen A). The main common feature is the presence of a strong dust lane, which is relatively straight and thin, crossing the luminous body along the minor axis. Apart from this dust lane, the luminous body possesses the smooth structure typical of the elliptical galaxies. Two other galaxies exhibiting the same morphology came immediately to our attention. They are NGC 1947 and to some extent, in spite of lack of resolution, the galaxy identified with Cyg A. We did not carry out any extended survey of this kind of galaxy, so the sample presented in this Letter (Fig. 1) is very limited. An additional galaxy identified with the radio source PKS 1934-63 must be included in the sample. Its optical similarity and radio similarity to NGC 5128 have recently been remarked by Penston and Fosbury (1978).

\section{MORPHOLOGICAL CONSIDERATIONS}

NGC 5128 and NGC 1947 are classified as lenticulars by de Vaucouleurs et al. NGC 5363, formerly classified Irr II (Holmberg 1958), was later also considered to be of class S0 (Krienke and Hodge 1974; Sandage and Visvanathan 1978). However, the galaxies discussed in this Letter are quite unlike the majority of S0 galaxies, in the sense that the main luminous body is systematically elongated in the direction perpendicular to the absorption feature.

In Figure 2 we give ellipticity profiles and position angles of the luminous component of the four galaxies considered. For Cyg A and NGC 5363 contact copies of the original plates were traced with the JoyceLoebl isodensitracer, while for NGC 1947 and NGC 5128 isophotes are from Sérsic (1968). A reasonable interpolation of the isophotes has been made where the absorption occurs. It appears that the elongation is not only typical of the outer regions but is also present in the inner ones.

Normal S0 galaxies with dust lanes, seen edge-on (see, e.g., NGC 4526, 4710, and 5866 in Sandage 1961), are characterized by a bulge which is flattened in the same direction as the disk in which the absorption feature lies. The facts that the dust lane in the galaxies discussed in this Letter always occurs perpendicular to the bulge flattening and that it is observed in a number of objects suggest that from the morphological point of view we may be dealing with a new type of galaxy and not simply with isolated cases of peculiar objects.

If we attempt a three-dimensional interpretation, we can argue that the relatively thin dark lane defines a plane seen edge-on symmetrically cutting the main luminous body of the galaxy. If one maintains the same picture as for S0 galaxies, i.e., a disk producing absorption on a bulge, one has to conclude that the luminous body in the present sample of galaxies possesses a prolate configuration. In the case of NGC 5128 the plane is well defined in the inner part of the galaxy, but shows a twisting effect in the outermost regions, resembling the warped structures observed in the disks of some spiral galaxies. In general, it will be difficult to see the dust lane if the plane in which it lies does not contain the line of sight, because of its relative thinness. Assuming that the tilt angle with respect to the line of sight of the gaseous and dust plane in the galaxies presented is about $10^{\circ}$, we deduce that the expected number of similar objects seen at different tilt angles is 5 times as great. Therefore we 
PLATE L10

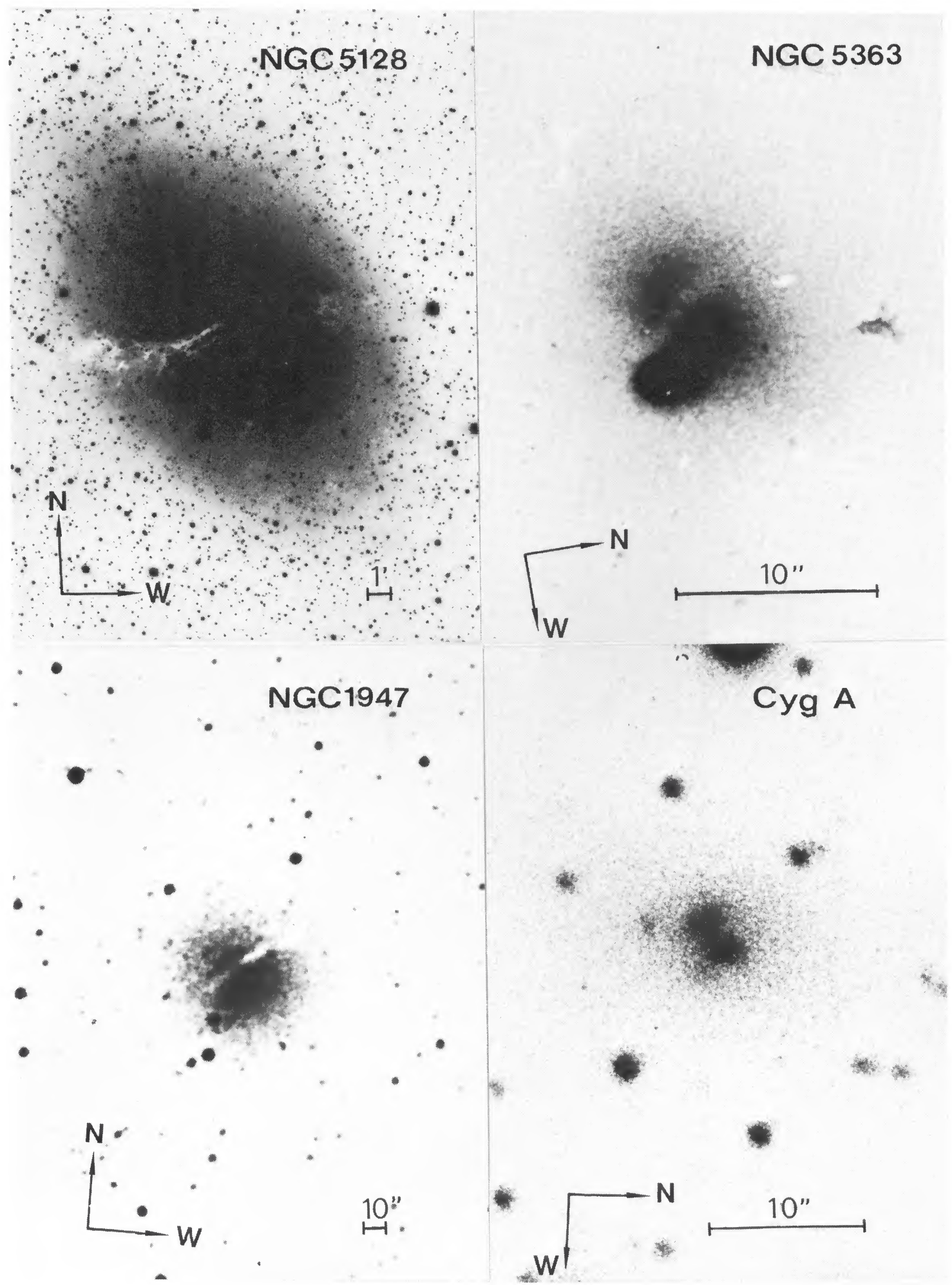

Fig. 1.- Photographs of NGC 5128, 5363, 1947, and Cyg A, showing the elongated structure crossed along the minor axis by a dust lane. NGC 5128 is from a deep IIIa-J plate taken with the $4 \mathrm{~m}$ CTIO telescope by Graham; NGC 5363 is from a plate taken by Hubble with the 100 inch $(2.54 \mathrm{~m})$ Hooker telescope; NGC 1947 is from the ESO B Atlas; and Cyg A is from a plate taken by Baade with the 200 inch $(5.08 \mathrm{~m})$ Hale telescope.

Bertola And Galletta (see page L115) 


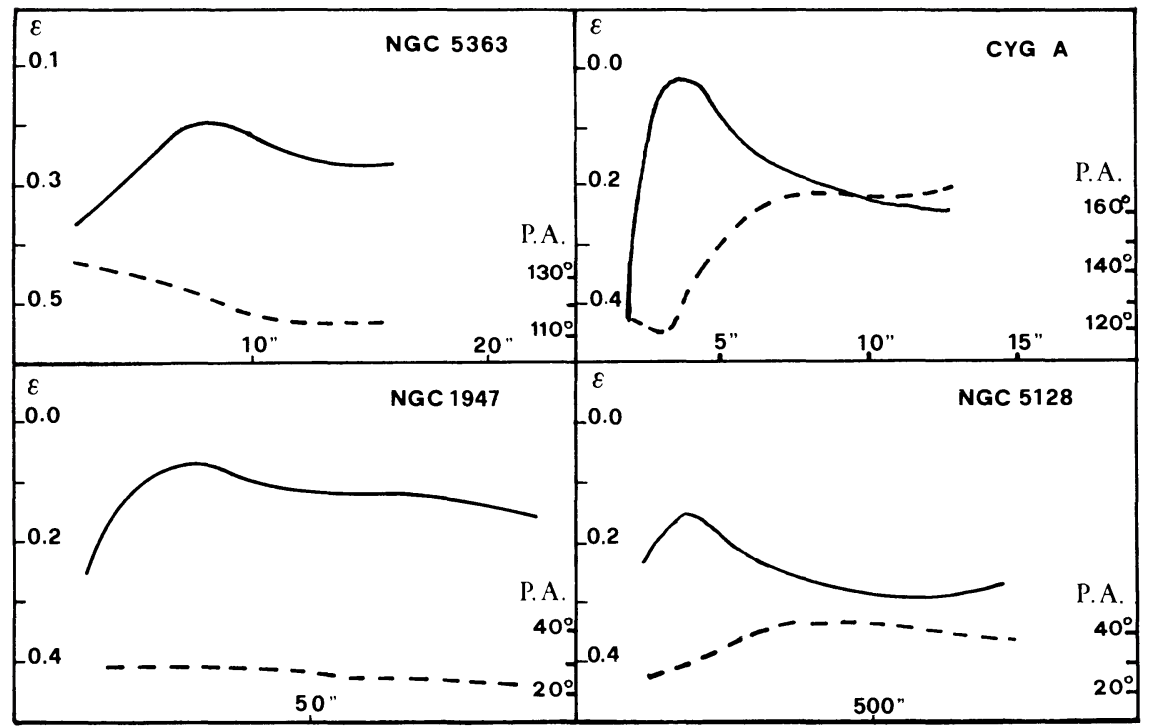

Fig. 2.-Ellipticity profiles (solid line) and position angle of the major axes (dashed line) as a function of the distance from the geometrical center of the isophotes.

conclude that this type of galaxy may comprise a significant number of objects. Similar galaxies with the plane seen edge-on would appear as normal ellipticals, with possible absorption features present where the dust is optically thick enough.

\section{DYNAMICAL CONSIDERATIONS}

The dynamical characteristics of this class of objects are complex and not clearly understood. According to Graham (1977) the gaseous disk of NGC 5128 rotates about the major axis, while an upper limit of only 30 $\mathrm{km} \mathrm{s}^{-1}$ at a distance of $1.5 \mathrm{kpc}$ in any direction can be set to the rotation of the elliptical component. Spectra of Cygnus A taken by Simkin (1977) show a similar dynamical behavior as far as the emission lines are concerned. No dynamical information is available for NGC 1947.

A 16 minute exposure high-resolution spectrum (N640b) of NGC 5363 has been obtained by one of us (F. B.) using the RC spectrograph attached to the Kitt Peak $4 \mathrm{~m}$ Mayall reflector, giving a dispersion of $54 \AA \mathrm{mm}^{-1}$ and a scale of $27^{\prime \prime} \mathrm{mm}^{-1}$. The slit was set at position angle $130^{\circ}$, which was judged the direction of the major axis on the Palomar Sky Survey print. The spectrum covers the region redward of $5800 \AA$ in the first order and it shows the following lines: [S II] $\lambda \lambda 6717-6731, \mathrm{H} \alpha,[\mathrm{N}$ II] $\lambda \lambda 6548-6584,[\mathrm{O}$ I] $\lambda 6300$, and the absorption doublet $\mathrm{Na}$ I $\lambda \lambda 5890-5896$, which is resolved. In the second order [O II] $\lambda \lambda 3727-3729$ is seen weakly. The ratio $\mathrm{H} \alpha / \mathrm{N}$ II is less than 1 , as is usually observed in the nuclear regions of galaxies. In Figure 3 we present the results obtained from measuring both the absorption and the emission lines. They were measured on isophotal tracings of the original spectrum obtained by using the Joyce-Loebl isodensitracer with enlargement of 100 times. This method has been proved convenient when dealing with spectra of small extensions normal to the dispersion. The error of mea- surement of a single point, as referred to the NW condensation, whose velocity is assumed as the zero point, is estimated $\pm 15 \mathrm{~km} \mathrm{~s}^{-1}$.

Over a measurable range of 6 " the absorption lines show a gradient of about $17 \mathrm{~km} \mathrm{~s}^{-1} \operatorname{arcsec}^{-1}$, the SE region being the receding one. A completely different behavior is exhibited by the emission lines, which are very strong on the NW side and extend faintly toward SE. In this connection it is interesting to note that van den Bergh (1976b) and Kronberg, van den Bergh, and Button (1977) have found similar characteristics in the optical counterpart of Cyg A, where the NW part is dominated by emission lines.

The velocity derived from the emission lines in NGC 5363 goes up to $50 \mathrm{~km} \mathrm{~s}^{-1}$ at $3^{\prime \prime}$ from the NW condensa-

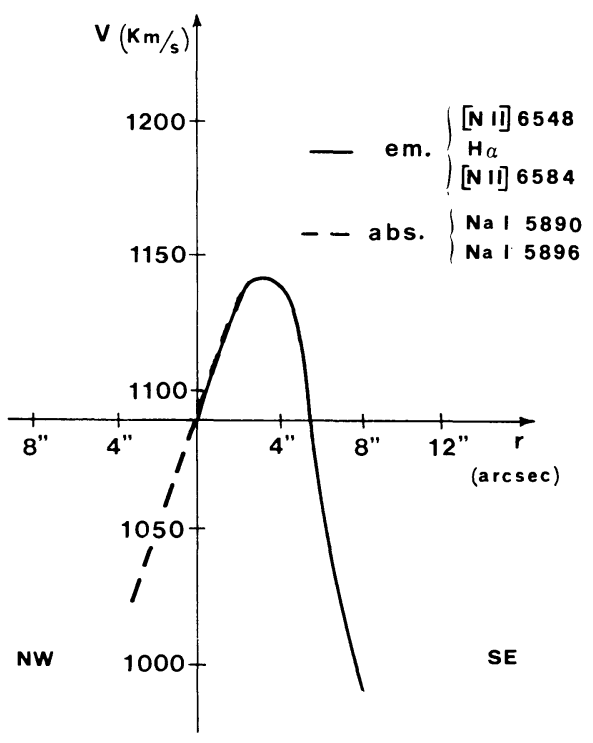

FIG. 3-The velocity field in NGC 5363 as measured from the emission and absorption lines. 
tion. It then decreases rapidly down to a value greater than $-100 \mathrm{~km} \mathrm{~s}^{-1}$. The velocity of recession, corrected for solar motion, is $1090 \mathrm{~km} \mathrm{~s}^{-1}$, in good agreement with previous determinations (de Vaucouleurs et al.). The conclusion we can draw from these meager data is that the dynamics of the stellar and gaseous components differ greatly.

\section{DISCUSSION}

The peculiarity of NGC 5128 has been such that in the past it has stimulated the search for galaxies with similar morphological structures and has been the subject of some controversy (de Vaucouleurs 1953; Baade and Minkowski 1954b). However, the morphological similarities which have been pointed out in this Letter, e.g., the elongated shape of the luminous component cut by a dust lane along the minor axis, seem to be well defined.

The fact that elliptical galaxies could be represented by prolate spheroids or triaxial ellipsoids has been recently emphasized by several authors. Gott and Thuan (1976) studied the collapse of a pure star protogalaxy, which in the early phases assumes alternately an oblate and a prolate configuration rotating along the minor and major axes, respectively. Miller (1978) has produced numerical models of elliptical galaxies which assume prolate stable configuration rotating end over end. The more general case of triaxial ellipsoidal configurations has been invoked by Binney (1978) to explain the low rotational velocity found in elliptical galaxies (Bertola and Capaccioli 1975; Illingworth 1977). Variations of the direction of the major axis of the isophotes in elliptical galaxies (Williams and Schwarzschild 1978; Bertola and Galletta 1978; Barbon, Benacchio, and Capaccioli 1978) support the idea of the presence of triaxial configurations.

An alternative explanation of the elongated optical shape is that it is due to explosive events, as suggested by the presence of strong extended radio emission in NGC 5128 and Cyg A lying in the same direction as the elongation (Arp 1978).

Van den Bergh (1976a) has suggested that NGC 5128 is a field elliptical galaxy which is rich in dust and gas because of the absence of interaction with the intergalactic medium. In the frame of this interpretation, we deduce from the occurrence of similar structures that the dust and gas are always concentrated in the same plane and that they are not distributed randomly. This obviously should be due to a dynamical effect. At the same time we deduce the existence of elliptical galaxies with prolate structures. In fact, it would be difficult to conceive of an oblate spheroidal structure cut by a dust plane perpendicular to the equatorial plane, because of a lack of cylindrical symmetry. The latter case could be explained only under very peculiar conditions, and their occurrence is not likely for every one of the objects presented here. The dynamical data available for NGC 5128 and Cyg A, showing a rotation of the gas along the major axis, give support to this interpretation. On the contrary, since the absorption lines in NGC 5363 seem to indicate a rotation of \pm 50 $\mathrm{km} \mathrm{s}^{-1}$ with the slit perpendicular to the presumed absorption disk, the disk would be tumbling and not spinning. However, the meager data available for NGC 5363, and especially the lack of spectra along the dust lane, do not permit a dynamical model.

NGC 5128, NGC 1947, and NGC 5363 are field galaxies, while Cyg $\mathrm{A}$ is one of the brightest members in a cluster (Baade and Minkowski 1954a). In the van den Bergh picture, we can explain the presence of gas in Cyg A because, if it lies at the center of mass of the cluster, the ram pressure is not effective on it.

Three of the objects presented are associated with double radio sources (Cyg A, Cen A, and PKS 193463), while NGC 5363 is connected with an unresolved source with a flat spectrum (Tovmasyan 1967). NGC 1947 is not found to be a radio source. We have not included in our sample NGC 1316, identified with the double radio source For A, since its optical morphology is somewhat complicated. However, there are resemblances which should be borne in mind.

The fact that three of five galaxies are strong double radio sources - a much greater fraction than the fraction of all galaxies which have these radio properties-indicates that we are dealing with a type of galaxy which may produce double radio sources with high frequency. At this point we ask ourselves whether many other radio galaxies are of this type but not recognizable because of the problem of detecting narrow dust lanes if the disk is not observed edge-on.

The range of absolute magnitudes of our sample is quite wide, from about -18 for NGC 1947 to -22 for Cyg A.

In connection with the presence of prolate structure, we would like to draw attention to NGC 2685 (Sandage 1961) and NGC 4650 (Sérsic 1967; Laustsen 1978) in the Centaurus I cluster, which, although their morphology is in some ways different from that of the present sample, exhibit an elongated structure perpendicular to a possible dust and gas plane. In these two galaxies this plane extends well outside along the minor axis of the luminous body. Because of these different morphological aspects we are not inclined to include these objects among the class defined in this Letter. However, it is quite possible that from the physical point of view we are dealing with similar formation processes.

Another case which we would like to mention in the context of this Letter is NGC 4278, where the stellar component has a major axis about perpendicular to the major axis defined by the neutral hydrogen (Knapp, Kerr, and Williams 1978).

The spectroscopic observations discussed in this Letter were carried out when one of us (F. B.) was Visiting Astronomer at Kitt Peak National Observatory. Access to part of the photographic material was possible when F. B. was Guest Observer at the Hale Observatories. It is a pleasure to thank J. A. Graham for permission to publish his photograph of NGC 5128.

We thank also M. Capaccioli, M. S. Longair, J. W. Sulentic, and S. van den Bergh for helpful discussions.

This work was partially supported by CNR under the Italy-USA Cooperative Science Program. 


\section{REFERENCES}

Arp, H. 1978, private communication.

Baade, W., and Minkowski, R. 1954a, A p.J., 119, 206.

- 1954b, Observatory, 74, 130.

Barbon, R., Benacchio, L., and Capaccioli, M. 1978, private copomunication.

Bertola, F., and Capaccioli, M. 1975, A p. J., 200, 439.

Bertola, F., and Galletta, G. 1978, in preparation.

Binney, J. 1978, M.N.R.A.S., 183, 561.

de Vaucouleurs, G. 1953, Observatory, 73, 252.

de Vaucouleurs, G., de Vaucouleurs, A., and Corwin, H. G. 1976, Second Reference Catalogue of Bright Galaxies (Austin: University of Texas Press)

Gibson, D. M. 1975, Astr. Ap., 39, 377.

Gott, J. R., and Thuan, T. X. 1976, A p. J., 204, 649.

Graham, J. A. 1977, Bull. A AS, 9, 630.

Holmberg, E. 1958, Medd. Lunds Astr. Obs., 2, 136

Illingworth, G. 1977, Ap. J. (Letters), 218, L43

Laustsen, S. 1978, ESO Annual Rept., 1977, p. 16.
Knapp, G. R., Kerr, F. J., and Williams, B. A. 1978, $A$ p. J., 222, 800.

Krienke, K., Jr., and Hodge, P. W. 1974, A.J., 79, 1242.

Kronberg, P., van den Bergh, S., and Button, S. 1977, A.J., 82, 315.

Miller, R. H. 1978, Ap. J., 223, 122.

Moffet, A. T. 1966, Ann. Rev. Astr. Ap., 4, 145.

Penston, M. W., and Fosbury, R. A. 1978, M.N.R.A.S., 183, 479.

Sandage, A. 1961, The Hubble Atlas of Galaxies (Washington: Carnegie Institution).

Sandage, A., and Visvanathan, N. 1978, Ap. J., 223, 707.

Sérsic, J. L. 1967, Zs. A p., 67, 306. servatory)

Simkin, S. M. 1977, A p. J., 217, 45

Tovmasyan, G. M. 1967, Astrofizika, 3, 427.

van den Bergh, S. 1976a, Ap. J., 208, 273.

- - 1976b, A p.J. (Letters), 210, L63.

Williams, T. B., and Schwarzschild, M. 1978, preprint.

Francesco Bertola and Giuseppe Galletta: Osservatorio Astronomico, 35100 Padova, Italy 